\title{
Magnetic control of convection in nonconducting paramagnetic fluids
}

\author{
Jie Huang and Boyd F. Edwards \\ Department of Physics, West Virginia University, P.O. Box 6315, Morgantown, West Virginia 26506-6315 \\ Donald D. Gray \\ Department of Civil and Environmental Engineering, West Virginia University, P.O. Box 6103, \\ Morgantown, West Virginia 26506-6103
}

(Received 25 April 1997)

\begin{abstract}
An inhomogeneous magnetic field exerts a magnetic body force on magnetically permeable fluids. A recent experiment [D. Braithwaite, E. Beaugnon, and R. Tournier, Nature (London) 354, 134 (1991)] demonstrates that this force can be used to compensate for gravity and to control convection in a paramagnetic solution of gadolinium nitrate. We provide the theory of magnetically controlled convection in a horizontal paramagnetic fluid layer heated from either above or below. Our theoretical predictions agree with the experiments. [S1063-651X(98)50901-2]
\end{abstract}

PACS number(s): 47.20.Bp, 47.27.Te, 47.62.+q

Recent experiments [1,2] observe the strong enhancing and suppressing effects of an applied inhomogeneous magnetic field on thermal transport in a gadolinium nitrate solution confined in a closed cylindrical cell heated from below, indicating that the thermal-gradient-induced buoyancydriven convection in this paramagnetic fluid is controllable via the applied magnetic field. We provide here the theory of magnetically controlled convection in a horizontal, nonconducting paramagnetic fluid layer heated from either above or below. We show that the convective fluid flow can be effectively controlled by placing the layer in a nonuniform magnetic field, which can promote or inhibit convection for both upward and downward thermal gradients. It has a great potential to be utilized to enhance or to suppress the gravitational effect in terrestrial experiments and to control the flow of nonconducting paramagnetic fluids in a microgravity environment. This effect might also be utilized to increase the efficiency of heat-transfer devices or to control microstructures in crystal growth from paramagnetic liquids. Our theoretical predictions agree with the experiments.

According to Landau and Lifshitz [3], magnetic forces per volume on a fluid in a magnetic field $\mathbf{H}$ are [Eq. (34.3) in Ref. [3] converted to SI units]

$$
\mathbf{f}=-\boldsymbol{\nabla} p_{0}+\frac{1}{2} \boldsymbol{\nabla}\left[H^{2} \rho\left(\frac{\partial \mu}{\partial \rho}\right)_{T}\right]-\frac{H^{2}}{2} \boldsymbol{\nabla} \mu+\mu \mathbf{j} \times \mathbf{H},
$$

where $p_{0}$ is the pressure in the absence of the field, $\rho$ the density of the fluid, $T$ the temperature, $\mu$ the magnetic permeability of the fluid, and $\mathbf{j}$ the electric current density in the fluid. For electrically nonconducting fluids, $\mathbf{j}=\mathbf{0}$ and therefore the last term vanishes. As $\mu=\mu_{0}(1+\chi), \mathbf{M}=\chi \mathbf{H}$, and $\boldsymbol{\nabla} \times \mathbf{H}=0$, we can rewrite Eq. (1) as

$$
\mathbf{f}=-\boldsymbol{\nabla} p_{0}-\frac{\mu_{0}}{2} \boldsymbol{\nabla}\left[H^{2}\left(\frac{\partial(\chi v)}{\partial v}\right)_{T}\right]+\mu_{0} \mathbf{M} \cdot \boldsymbol{\nabla} \mathbf{H},
$$

where $\mu_{0}$ is the permeability of free space, $v=1 / \rho$ the specific volume, $\chi$ the volumetric susceptibility of the fluid, and $\mathbf{M}$ the magnetization (the magnetic moment per unit vol- ume). For pure paramagnetic fluids such as liquid oxygen, the magnetic susceptibility satisfies Curie's law [4], i.e., $\chi$ $=C \rho / T$, where $C$ is a constant characteristic of the fluid. The second term on the right-hand side of Eq. (2) therefore vanishes identically for such fluids. For paramagnetic fluids such as aqueous solutions of gadolinium nitrate or manganese chloride, the magnetic susceptibility is also proportional to the solute concentration and hence the second term does not vanish identically. However, since this term is a pure gradient, it only modifies the pressure and has no contribution to convection since $\boldsymbol{\nabla} \times \boldsymbol{\nabla} p=0$. The last term in Eq. (2) is the Kelvin body force [5] $\mathbf{f}_{m}=\mu_{0}(\mathbf{M} \cdot \boldsymbol{\nabla}) \mathbf{H}$, which arises from the interaction between the local magnetic field $\mathbf{H}$ within the fluid and the molecular magnetic moments characterized by the magnetization M. Additional dissipative forces on colloidal ferrofluids [6] are negligible in nonconducting paramagnetic fluids. An imposed thermal gradient produces a spatial variation in the magnetization through the temperature-dependent magnetic susceptibility and therefore renders the Kelvin body force density $\mathbf{f}_{m}$ nonuniform spatially. This thermal-gradient-induced inhomogeneous magnetic body force density $\mathbf{f}_{m}$ can promote or inhibit convection in a manner similar to the gravitational body force.

We consider an incompressible horizontal paramagnetic fluid layer of thickness $d$ defined by $|z|<d / 2$, with $\hat{\mathbf{z}}$ pointing up. This layer is heated from above or below with a temperature difference $\Delta T$ between the bottom and the top (positive for heating from below) and is subjected to an applied external nonuniform magnetic field with a constant field gradient $\mathbf{H}^{\text {ext }}=\mathbf{H}_{0}+(\mathbf{r} \cdot \boldsymbol{\nabla}) \mathbf{H}^{\text {ext }}$, where $\mathbf{r}=x \hat{\mathbf{x}}+y \hat{\mathbf{y}}+z \hat{\mathbf{z}}$ is the position vector. Here the vector $\mathbf{H}_{0}$ is the magnetic field at the center of the layer and the field gradient $\boldsymbol{\nabla} \mathbf{H}^{\text {ext }}$ is assumed to be a constant tensor. Maxwell's equations require this tensor to be symmetric and traceless.

The fluid flow is governed by the Navier-Stokes equations in addition to Maxwell's equations for the magnetic field $\mathbf{H}$ and magnetic induction $\mathbf{B} \equiv \mu_{0}(\mathbf{M}+\mathbf{H})$. The purely thermal conduction state is described by the static state solution of these equations. Adding departures to the static state and substituting the perturbed state into these equations yield the 
equations governing the convective flow. Under the Oberbeck-Boussinesq approximation, which allows small density gradients only in the gravity term of the NavierStokes equations, we write the dimensionless governing equations for the convective flow,

$$
\begin{aligned}
& \frac{1}{\operatorname{Pr}}\left(\frac{\partial \mathbf{v}}{\partial t}+\mathbf{v} \cdot \boldsymbol{\nabla} \mathbf{v}\right)=-\nabla p+\left(R \hat{\mathbf{z}}-\mathbf{R}_{m}\right) \theta+K \sin ^{2} \phi \theta \hat{\mathbf{z}} \\
& +K(z-\theta) \hat{\mathbf{H}}_{0} \cdot \boldsymbol{\nabla} \mathbf{h}+\nabla^{2} \mathbf{v} \\
& \left(\frac{\partial \theta}{\partial t}+\mathbf{v} \cdot \nabla \theta-\hat{\mathbf{z}} \cdot \mathbf{v}\right)=\nabla^{2} \theta+\Phi \\
& \boldsymbol{\nabla} \cdot \mathbf{h}-\hat{\mathbf{H}}_{0} \cdot \boldsymbol{\nabla} \theta=0, \quad \boldsymbol{\nabla} \cdot \mathbf{v}=0 .
\end{aligned}
$$

Here $\mathbf{v}, p, \theta$, and $\mathbf{h}$ represent the departures from the static state solution: velocity, pressure (including magnetic contributions), temperature, and magnetic field, respectively. In these equations $\hat{\mathbf{H}}_{0}=\mathbf{H}_{0} / H_{0}$ is the unit vector in the $\mathbf{H}_{0}$ direction, $\phi$ the angle between $\mathbf{H}_{0}$ and the horizontal, and $\Phi$ the viscous dissipation. Equation (3) involves the Prandtl number $\operatorname{Pr}=\nu / D_{T}$, the Rayleigh number $R$ $=\alpha g d^{3} \Delta T / \nu D_{T}$, the Kelvin number

$$
K=\frac{\mu_{0} \chi_{a}^{2} \Delta T^{2} d^{2} H_{0}^{2}}{\left(1+\chi_{a}\right) \rho_{a} T_{a}^{2} \nu D_{T}}
$$

and the magnetic vector control parameter

$$
\mathbf{R}_{m}=\frac{\mu_{0} \chi_{a} d^{3} \Delta T}{\rho_{a} T_{a} \nu D_{T}} \mathbf{H}_{0} \cdot \boldsymbol{\nabla} \mathbf{H}^{\mathrm{ext}}
$$

where $\nu$ is the kinematic viscosity, $D_{T}$ the thermal diffusivity, $\alpha$ the thermal expansion coefficient, $g$ the acceleration of gravity, $T_{a}$ the average temperature of the layer, $\chi_{a}$ the susceptibility at $T_{a}$, and $\rho_{a}$ the density at $T_{a}$.

In the presence of a uniform oblique magnetic field ( $K \neq 0$, but $\mathbf{R}_{m}=\mathbf{0}$ ), our analysis [7] shows that longitudinal rolls with axes parallel to the horizontal component of the field are the rolls most unstable to convection, reflecting the breaking of the rotational symmetry of the layer about the vertical due to the horizontal component of the field. The corresponding critical Rayleigh number and critical wavelength for the onset of such rolls are less than the wellknown Rayleigh-Bénard values [8] in the absence of magnetic fields.

The vector parameter $\mathbf{R}_{m}$ in Eq. (3) measures the strength of the magnetic body force due to the applied field gradient. The combination of the vertical component of $\mathbf{R}_{m}$ with $R$ in Eq. (3) shows that the gravitational effect on the convective flow can be balanced by this component of $\mathbf{R}_{m}$. Therefore, convection in nonconducting paramagnetic fluids can be controlled by an inhomogeneous magnetic field. Furthermore, the curl of Eq. (3) indicates that convection in a horizontal plane is possible if $\mathbf{R}_{m}$ has a nonzero horizontal component. On the contrary, such convection is impossible in the nonmagnetic case or in a uniform applied field.

We now apply the theory to the experiments $[1,2]$ to illustrate the controlling effect of a nonuniform magnetic field on convection in paramagnetic fluids. In these experiments, a closed cylindrical convection cell is placed in the central area near the end of a superconducting coil. Within the small area of the cell, the magnetic field produced by the coil is approximated by $\mathbf{H}=H_{0} \hat{\mathbf{z}}-H_{1} x \hat{\mathbf{x}}-H_{1} y \hat{\mathbf{y}}+2 H_{1} z \hat{\mathbf{z}}$, where the parameters $H_{0}$ and $H_{1}$ are constants determined by the geometrical properties of the coil. This external magnetic field produces a magnetic control parameter $\mathbf{R}_{m}=R_{m} \hat{\mathbf{z}}$, where

$$
R_{m}=\left.\frac{\mu_{0} \chi_{a} d^{3} \Delta T}{\rho_{0} T_{a} \nu D_{T}} H \frac{\partial H}{\partial z}\right|_{\mathbf{r}=\mathbf{0}}
$$

We use rigid (no-slip) boundary conditions, ignore sidewall effects, and demand continuity of the normal component of magnetic induction and the tangential component of magnetic field across the top and bottom boundaries. A linear stability analysis using the Galerkin method [7] then yields the critical temperature difference $\Delta T_{c}$ for the critical state for given $H$ and $H(\partial H / \partial z)$. These results are summarized in the equation

$$
\frac{d^{3} \Delta T_{c}}{\nu D_{T}}\left[\alpha g+\frac{0.665 \mu_{0} \chi_{a}^{2} \Delta T_{c} H^{2}}{\left(1+\chi_{a}\right) \rho_{a} T_{a}^{2} d}-\frac{\mu_{0} \chi_{a}}{\rho_{a} T_{a}} H \frac{\partial H}{\partial z}\right]=R_{c},
$$

which agrees with the numerical results to within $1 \%$ over the entire parameter range. Here the universal numbers $R_{c}$ $\approx 1708$ and 0.665 arise from the numerical calculation and are independent of any fluid properties. Convection sets in for $\Delta T \geqslant \Delta T_{c}$. The critical wave number for the onset of convection is shifted by the uniform magnetic field [7], but is unaffected by the field gradient.

In experiments $[1,2]$, the value of the volumetric susceptibility is $\chi=1.63 \times 10^{-4}$ at room temperature and the depth of the cell is $d=1.0 \times 10^{-2} \mathrm{~m}$. We use the values for parameters $\rho_{a}, \alpha, \nu$, and $D_{T}$ for water to analyze the experiments due to the lack of available values for the gadolinium nitrate solution. In the absence of magnetic fields, buoyancy is the only driving force for convection and Eq. (7) yields $\Delta T_{c}$ $\sim 1{ }^{\circ} \mathrm{C}$. A calculation of the ratio of the second term to the third term in Eq. (7) for these experiments shows that the second term can be neglected.

Equation (7) shows that the effect of the magnetic force on convection depends on the direction of the force. When the cell is heated from above, $\Delta T<0$, gravity tends to stabilize the fluid. When the applied magnetic force is upward, $H(\partial H / \partial z)>0$, this magnetic force tends to destabilize the fluid and to promote convection. Equation (7) shows that when $\mu_{0}^{2} H(\partial H / \partial z) \leqslant \mu_{0} \alpha g \rho_{a} T_{a} / \chi_{a}=5.3 \mathrm{~T}^{2} / \mathrm{m}$, the fluid is stable to any perturbations. This result is independent of the thermal diffusivity and kinematic viscosity of the fluid. The experiment [2] observed that when $\mu_{0}^{2} H(\partial H / \partial z) \leqslant 5 \mathrm{~T}^{2} / \mathrm{m}$, the Nusselt number, the ratio of the heat transport with and without convection, remains unity for $\Delta T$ up to $32{ }^{\circ} \mathrm{C}$ (see Fig. 3a in Ref. [2]), indicating no convection, consistent with the theory. When $\mu_{0}^{2} H(\partial H / \partial z) \geqslant 6 \mathrm{~T}^{2} / \mathrm{m}$, the Nusselt number exceeds unity for small $\Delta T$, indicating the onset of magnetic convection as predicted. The Nusselt number increases with increasing $H(\partial H / \partial z)$ for given $\Delta T$.

When the cell is heated from below and the magnetic force is downward, Eq. (7) shows that the magnetic field enhances convection. The measured Nusselt number in- 
creases as $|H(\partial H / \partial z)|$ increases for any $\Delta T$ (see Fig. 2a in Ref. [1] and Fig. 3c in Ref. [2]), reflecting the enhancement of convection. When the magnetic force is upward, Eq. (7) implies that the magnetic field reduces convection for $\mu_{0}^{2} H(\partial H / \partial z)<5.3 \mathrm{~T}^{2} / \mathrm{m}$ and prevents convection for $\mu_{0}^{2} H(\partial H / \partial z) \geqslant 5.3 \mathrm{~T}^{2} / \mathrm{m}$. The experiment observed that when $\mu_{0}^{2} H(\partial H / \partial z) \leqslant 5 \mathrm{~T}^{2} / \mathrm{m}$, the Nusselt number decreases as $H(\partial H / \partial z)$ increases for all $\Delta T$, reflecting the suppressing effect as predicted (see Fig. 2b in Ref. [1] and Fig. 3b in Ref. [2]). When $\mu_{0}^{2} H(\partial H / \partial z)=6 \mathrm{~T}^{2} / \mathrm{m}$, the measured Nusselt number remains unity for $\Delta T \leqslant 5{ }^{\circ} \mathrm{C}$, indicating no convection, consistent with the theory. However, the Nusselt number begins to increase for $\Delta T>5^{\circ} \mathrm{C}$, indicating the presence of convection in contrast with the theory. This discrepancy might be due to the large size of the cell (diameter $25 \mathrm{~mm}$ ) compared to the coil (diameter $120 \mathrm{~mm}$ ); the magnetic force near the sidewall of the cell might not be strong enough to overwhelm the buoyancy. The measured Nusselt number re- mains unity for all $\Delta T$ for $\mu_{0}^{2} H(\partial H / \partial z)=15 \mathrm{~T}^{2} / \mathrm{m}$, indicating the complete suppression of convection. Our analysis ignores Soret effects; the good agreement with the experimental results indicates that Soret effects may be negligible in these experiments.

In conclusion, the convective fluid flow in nonconducting paramagnetic fluids can be effectively controlled by an external inhomogeneous magnetic field. The provided theory of magnetically controlled convection in paramagnetic fluids shows that convection in such fluids is controlled by the applied magnetic field through the vector parameter $\mathbf{R}_{m}$. The gravitational effect can be balanced by the field. This magnetic effect can be utilized to enhance or to suppress gravitational convection in terrestrial experiments and to provide an effective body force in a microgravity environment.

This research was supported by NASA under Grant No. NAG3-1921.
[1] D. Braithwaite, E. Beaugnon, and R. Tournier, Nature (London) 354, 134 (1991).

[2] E. Beaugnon et al., J. Phys. I 3, 399 (1993).

[3] L. D. Landau and E. M. Lifshitz, Electrodynamics of Continuous Media (Pergamon, Oxford, 1960), Chap. 4.

[4] N. W. Ashcroft and N. D. Mermin, Solid State Physics (Saunders, Philadelphia, 1976), Chap. 31.
[5] R. E. Rosensweig, Ferrohydrodynamics (Cambridge University Press, New York, 1985), Chap. 4.

[6] M. Liu, Phys. Rev. Lett. 74, 4535 (1995).

[7] J. Huang, B. F. Edwards, and D. D. Gray, Phys. Fluids 9, 1819 (1997).

[8] S. Chandrasekhar, Hydrodynamic and Hydromagnetic Stability (Dover, New York, 1981), Chap. 2. 